\title{
A Signal Processing Algorithm Based on Parametric Dynamic Programming
}

\author{
Andrey Kopylov ${ }^{1}$, Olga Krasotkina ${ }^{1}$, Oleksandr Pryimak ${ }^{2}$, and Vadim Mottl ${ }^{3}$ \\ ${ }^{1}$ Tula State University, Lenin pr. 92, 300600 Tula, Russia \\ ${ }^{2}$ Moscow Institute of Physics and Technology, 141700, 9, Institutskii per., Dolgoprudny, \\ Moscow Region, Russia \\ ${ }^{3}$ Dorodnicyn Computing Centre of RAS, Vavilov st. 40, 119333 Moscow, Russia \\ \{And.Kopylov, aleksandr.priymak\} @gmail.com, \\ $\{$ ko180177, vmottl\} @yandex.ru
}

\begin{abstract}
A new algorithm for low-level signal processing is proposed based on dynamic programming principle. It is shown that it is possible to extend the dynamic programming procedure to the case of continuous variables by introducing the parametric family of Bellman functions, represented as a minimum of a set of quadratic functions. The procedure can take into account a wide range of prior assumptions about the sought-for result, and leads to the effective algorithms of data analysis.
\end{abstract}

Keywords: dynamic programming, edge-preserving smoothing, separable optimization.

\section{Introduction}

There is a considerably wide class of low-level signal processing problems, where the optimization-based approach is claimed to play a role of the universal framework. This class includes, in particular, such data analysis problems as smoothing, nonstationary regression analysis, segmentation, etc.

In all these cases the ultimate aim of processing can be represented as a transformation of the original data $Y=\left(y_{\mathbf{t}}, \mathbf{t} \in T\right), y \in \mathrm{Y}$, defined on a subset $T$, of the signal axis, into a secondary array $X=\left(x_{\mathbf{t}}, \mathbf{t} \in T\right)$ which would be defined on the same argument set $\mathbf{t} \in T$ and take values $x \in \mathrm{X}$ from a set specific for each particular problem. It is important that the signal axis is naturally supplied by the binary neighborhood relation, which turns it into a simple graph in the form of a chain.

The problem of finding the best, in some sense, transformation of source data $Y$ into a secondary array $X$ can be mathematically set as those of minimizing objective functions $J(X)$ of a special kind, defined over the variety of all the feasible results of processing, and meant to assess the discrepancy between each admissible version of the result $X$ and the given data array $Y$.

$$
J(X)=\sum_{\mathbf{t} \in T} \psi_{\mathbf{t}}\left(x_{\mathbf{t}} \mid Y_{\mathbf{t}}\right)+\sum_{\left(\mathbf{t}^{\prime}, \mathbf{t}^{\prime \prime}\right) \in G} \gamma_{\mathbf{t}^{\prime}, \mathbf{t}^{\prime \prime}}\left(x_{\mathbf{t}^{\prime}}, x_{\mathbf{t}^{\prime \prime}}\right)
$$


The structure of objective function $J(X)$ reflects the fact that the data are ordered along the axis of one or two arguments, and can be defined by the undirected neighborhood graph $G \subset T \times T$. Such objective functions are called pair-wise separable, as far as they are representable as sums of elementary objective functions each of only one or two variables. These functions are associated, respectively, with nodes and edges of the neighborhood graph.

The data dependent node functions $\psi_{\mathbf{t}}\left(x_{\mathbf{t}} \mid Y_{\mathbf{t}}\right)$ are to be chosen with respect to the essence of a particular data processing problem so that the greater value each of them takes, the more evident is the contradiction between the hypothesis that $x_{t}$ is just the correct local value we are seeking and the respective vicinity $Y_{\mathbf{t}}$ of the data array. Each of the model based edge functions $\gamma_{\mathbf{t}^{\prime}, \mathbf{t}^{\prime \prime}}\left(x_{\mathbf{t}^{\prime}}, x_{\mathbf{t}^{\prime \prime}}\right)$ is meant to impose an individual penalty upon the distinction of values in the respective pair of adjacent variables of the edge $\left(\mathbf{t}^{\prime}, \mathbf{t}^{\prime \prime}\right)$ of neighborhood graph $G$. The evident neighborhood graph $G$ for signals is chain.

In the case of finitely valued objective variables $x_{\mathbf{t}} \in \mathrm{X}=\{1, \ldots, m\}$, such an optimization problem is known under the name of the $(\min ,+)$ labeling problem. For an arbitrary adjacency graph $G$ this problem is NP-hard, however, in the case of acyclic graph it is possible to construct very effective optimization procedures, dynamic programming (DP) in nature. But if the number of elements in the set $X$ grows, the computation time and the amount of computer memory for large majority of $(\min ,+)$ optimization algorithms becomes inapplicable very fast.

Nevertheless, it is possible to construct a generalization of the classical dynamic programming procedure to the case of continuous variables [1, 2]. Such a generalization can be made on the basis of introducing the concept of a parametric family of Bellman functions.

It will allow us to reduce, first, requirements to memory, as the number of parameters of Bellman functions is less than the number of elements in the set $X$, second, to increase computational speed, as we can turn the intermediate optimization problems on each step of the DP procedure to recurrent reevaluation of parameters, third, to increase accuracy in the tasks, where objective variables continuous in nature. Unfortunately, the class of appropriate parametric families of functions is quite deficient, and greatly reduces the amount of practical applications of parametric DP procedures.

We propose here a way to extend parametric family of Bellman functions to the case of most widely used optimization criteria

\section{Generalized Dynamic Programming Procedure}

A pair-wise separable objective function (1) allows a highly effective global optimization procedure [1] when the neighborhood graph on the set of its variables has no cycles, i.e. is a tree (Fig. 1). 


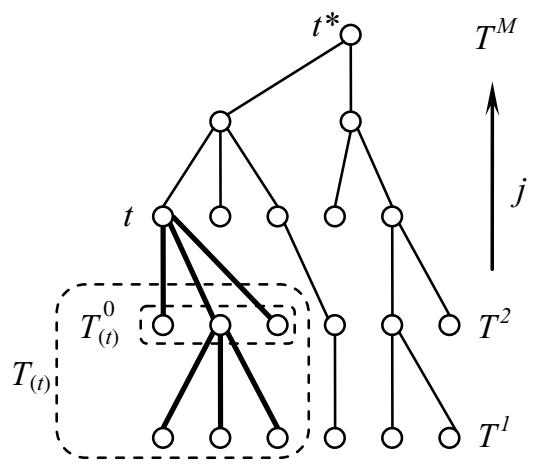

Fig. 1. Structure of an arbitrary tree

The optimization procedure in this case is based on a recurrent decomposition of the initial optimization problem of a function of $|T|$ variables, where $|T|$ is the number of elements in set $\mathrm{T}$, into a succession of $|T|$ elementary problems, each of which consists in optimization of a function of only one variable. The elementary functions of one variable to be minimized at each step of minimization of a treesupported separable function play the same role as the Bellman functions in the classical procedure and are called here extended Bellman functions.

The fundamental property of the Bellman function

$$
\tilde{J}_{t}\left(x_{t}\right)=\psi_{t}\left(x_{t}\right)+\sum_{s \in T_{(t)}^{0}} \min _{x_{s} \in \mathrm{X}}\left\{\gamma_{t, s}^{m}\left(x_{t}, x_{s}\right)+\tilde{J}_{s}\left(x_{s}\right)\right\}
$$

will be called the upward recurrent relation. The inverted form of this relation

$$
\tilde{x}_{s}\left(x_{t}\right)=\underset{x_{s} \in \mathrm{X}}{\arg \min }\left\{\gamma_{t, s}^{m}\left(x_{t}, x_{s}\right)+\tilde{J}_{s}\left(x_{s}\right)\right\}, s \in T_{(t)}^{0}
$$

will be referred to as the downward recurrent relation.

The procedure runs through all the nodes of the graph in order of their membership in the hierarchy levels $T^{j}$ at the upward pass $j=1, \ldots, M$, recurrently calculate and store Bellman functions in accordance with upward recurrent relation (2) starting with $\tilde{J}_{t}(x)=\psi_{t}\left(x_{t}\right), x \in \mathrm{X}, t \in T^{1}$. The Bellman function at the root $\tilde{J}_{t^{*}}(x), x \in \mathrm{X}$, that is obtained at the last step of the upward pass, immediately gives the optimal value of the root variable. On the downward pass, as the procedure descends the hierarchy levels $j=M, \ldots, 2$, the already found optimal values of the current level determine optimal values of the variables at the immediately underlying level in accordance with the downward recurrent rule (3).

In the case of continuous variables, e.g. if $\mathbf{X}=\left(x_{\mathbf{t}}, \mathbf{t} \in T\right), x_{\mathbf{t}} \in \mathrm{R}^{\mathrm{n}}$, a numerical realization of the basic procedure for a separable function supported by a tree, as well as the classical dynamic programming procedure, is possible only in case there exists 
a finitely parameterized function family $\tilde{J}(x, \mathbf{a})$ concordant with node functions $\psi_{\mathbf{t}}(x)$ and edge functions $\gamma_{\mathbf{t}^{\prime} \mathbf{t}^{\prime \prime}}\left(x_{\mathbf{t}^{\prime}}, x_{\mathbf{t}^{\prime \prime}}\right)$ in the sense that Bellman functions $\tilde{J}_{\mathbf{t}}\left(x_{\mathbf{t}}\right)$ belong to this family at each step. In this case, the upward pass of the procedure consists in recurrent re-evaluating parameters $\tilde{\mathbf{a}}_{\mathbf{t}}$ that completely represent the Bellman functions. $\tilde{J}_{\mathbf{t}}(x)=\tilde{J}\left(x, \tilde{\mathbf{a}}_{\mathbf{t}}\right)$.

In particular, as is shown in [1], if the node and edge functions are quadratic, the Bellman functions will be quadratic too. In such case, the DP procedure is entirely equivalent to Kalman-Bucy filter and interpolator [3]. However, prior smoothness constraints in the form of quadratic edge functions do not allow to keep abrupt changes of the data in the course of processing.

The parametric representation is also possible in the case of using absolute value of difference of adjacent variables instead of quadratic node functions. It leads to piecewise constant form of sought-for data array [2]. Though this kind of assumption is appropriate for separate problems, for example in economy, it yields not so good result generally.

We propose here to use the following form of the edge functions in criterion (1):

$$
\gamma_{\mathbf{t}^{\prime}, \mathbf{t}^{\prime \prime}}\left(x_{\mathbf{t}^{\prime}}, x_{\mathbf{t}^{\prime \prime}}\right)=\min \left[\left(x_{\mathbf{t}^{\prime}}-x_{\mathbf{t}^{\prime \prime}}\right)^{T} U_{\mathbf{t}^{\prime}, \mathbf{t}^{\prime \prime}}\left(x_{\mathbf{t}^{\prime}}-x_{\mathbf{t}^{\prime \prime}}\right), h\right],\left(\mathbf{t}^{\prime}, \mathbf{t}^{\prime \prime}\right) \in G
$$

It has been known [4], that the following edge function possesses the properties to preserve the abrupt changes in the data being analyzed. The node functions are also chosen in the quadratic form $\psi_{\mathbf{t}}\left(x_{\mathbf{t}} \mid Y_{\mathbf{t}}\right)=\left(y_{\mathbf{t}}-x_{\mathbf{t}}\right)^{T} B_{\mathbf{t}}\left(y_{\mathbf{t}}-x_{\mathbf{t}}\right)$. In this case it is possible to construct the parametric DP procedure for minimization of the criterion in the form (1).

Let us consider the case, then the adjacency graph $G$ has a form of a chain, so the source data $\mathbf{X}=\left(x_{\mathbf{t}}, \mathbf{t} \in T\right)$ and the result of processing $Y=\left(y_{\mathbf{t}}, \mathbf{t} \in T\right)$ are signals and $\mathbf{t}=1,2, \ldots N$.

It can be proven, that if the Bellman function at the node $t-1$ is

$$
\tilde{J}_{t-1}\left(x_{t-1}\right)=\min \left[\tilde{J}_{t-1}^{(1)}\left(x_{t-1}\right), \tilde{J}_{t-1}^{(1)}\left(x_{t-1}\right), \ldots, \tilde{J}_{t-1}^{\left(K_{t-1}\right)}\left(x_{t-1}\right)\right],
$$

then the next Bellman function will be:

$$
\tilde{J}_{t}\left(x_{t}\right)=\min \left[\tilde{J}_{t}^{(1)}\left(x_{t}\right), \tilde{J}_{t}^{(1)}\left(x_{t}\right), \ldots, \tilde{J}_{t}^{\left(K_{t}\right)}\left(x_{t}\right)\right],
$$

where $K_{t}=K_{t-1}+1$ and, in accordance with (2),

$$
\begin{gathered}
\tilde{J}_{t}^{(i)}\left(x_{t}\right)=\psi_{t}\left(x_{t}\right)+\min _{x_{t-1} \in \mathrm{X}}\left\{\left(x_{t}-x_{t-1}\right)^{T} U_{t}\left(x_{t}-x_{t-1}\right)+\tilde{J}_{t-1}^{(i)}\left(x_{t-1}\right)\right\}, i=1,2, \ldots K_{t}-1, \\
\tilde{J}_{t}^{\left(K_{t}\right)}\left(x_{t}\right)=\psi_{t}\left(x_{t}\right)+\min _{x_{t-1} \in \mathrm{X}}\left\{h+\tilde{J}_{t-1}\left(x_{t-1}\right)\right\} .
\end{gathered}
$$

It is easy to see that each function $\tilde{J}_{t}^{(i)}\left(x_{t}\right)$ has the parametric representation 


$$
\tilde{J}_{t}^{(i)}\left(x_{t}\right)=\left(x_{t}-x_{t}^{(i)}\right)^{T} R_{t}^{(i)}\left(x_{t}-x_{t}^{(i)}\right)+d_{t}^{(i)}, i=1,2, \ldots K_{t} .
$$

The downward recurrent relation (3) takes the form

$$
\tilde{x}_{t-1}^{(i)}\left(x_{t}\right)=\underset{x_{t-1} \in \mathrm{X}}{\arg \min }\left\{\min \left[f_{t}^{(1)}\left(x_{t-1}, x_{t}\right), f_{t}^{(2)}\left(x_{t-1}, x_{t}\right), \ldots, f_{t}^{\left(K_{t-1}\right)}\left(x_{t-1}, x_{t}\right), f_{t}^{\left(K_{t}\right)}\left(x_{t-1}\right)\right]\right\},
$$

where

$$
\begin{gathered}
f_{t}^{(i)}\left(x_{t-1}, x_{t}\right)=\left(x_{t}-x_{t-1}\right)^{T} U_{t}\left(x_{t}-x_{t-1}\right)+\tilde{J}_{t-1}^{(i)}\left(x_{t-1}\right), i=1,2, \ldots K_{t}-1, \\
f_{t}^{\left(K_{t}\right)}\left(x_{t-1}\right)=h+\tilde{J}_{t-1}\left(x_{t-1}\right) .
\end{gathered}
$$

Let $\quad \tilde{J}_{t}\left(x_{t}\right)=\min \left[J_{t}^{(1)}\left(x_{t}\right), J_{t}^{(2)}\left(x_{t}\right) \ldots, J_{t}^{(K)}\left(x_{t}\right)\right]$. If for any $x_{t} \in R$, $J_{t}^{(1)}\left(x_{t}\right) \geq \min \left[J_{t}^{(2)}\left(x_{t}\right) \ldots, J_{t}^{(K)}\left(x_{t}\right)\right]$, then $\tilde{J}_{t}\left(x_{t}\right)=\min \left[J_{t}^{(2)}\left(x_{t}\right) \ldots, J_{t}^{(K)}\left(x_{t}\right)\right]$. Thus, the number of functions in the representation of Bellman function at the step $t$ can be reduced. We consider the procedure of such reduction in the following section by the example of the edge-preserving smoothing of signals.

\section{Parametric DP Procedure for Edge-Preserving Smoothing of Signals}

Let us suppose that the observable signal $Y=\left(y_{t}=x_{t}+\xi_{t}, t=1, \ldots, N\right), y \in R$ where $\xi$ - additive white Gaussian noise, with zero mean. The aim of processing is to restore the hidden signal $X=\left(x_{t}, t=1, \ldots, N\right), x_{t} \in R$. The basic assumption in the problem is that the original signal changes smoothly enough except, perhaps, some points, where jumps or breaks can be observed.

It has been known [4], that the following edge function possesses the required properties of edge-preservation:

$$
\gamma_{t-1, t}\left(x_{t-1}, x_{t}\right)=u \min \left[\left(x_{t-1}-x_{t}\right)^{2}, \Delta^{2}\right] .
$$

It is easy to see, that the form of function (5) coincides with (4), if it is remembered that constant is the degenerate form of a quadratic function. The criterion (1) takes the following simple form:

$$
J(X)=\sum_{t=1}^{N}\left(y_{t}-x_{t}\right)^{2}+u \sum_{t=2}^{N} \min \left[\left(x_{t-1}-x_{t}\right)^{2}, \Delta^{2}\right] .
$$

Suppose that in step $t-1$, the Bellman function $\tilde{J}_{t-1}\left(x_{t-1}\right)$ is represented in the form

$$
\tilde{J}_{t-1}\left(x_{t-1}\right)=\min \left[J_{t-1}^{(1)}\left(x_{t-1}\right), J_{t-1}^{(2)}\left(x_{t-1}\right) \ldots, J_{t-1}^{(K-1)}\left(x_{t-1}\right)\right],
$$

where $J_{t-1}^{(i)}\left(x_{t-1}\right)=c_{t-1}^{(i)}\left(x_{t-1}-x_{t-1}^{(i)}\right)^{2}+d_{t-1}^{(i)}, c_{t-1}^{(i)} \geq 0$. 
Then, in accordance with forward recurrent relation (2) of a DP procedure, the Bellman function $\tilde{J}_{t}\left(x_{t}\right)$ at the next step will be

$$
\begin{gathered}
\tilde{J}_{t}\left(x_{t}\right)=\min \left[J_{t}^{(1)}\left(x_{t}\right), J_{t}^{(2)}\left(x_{t}\right) \ldots, J_{t}^{(K)}\left(x_{t}\right)\right], \\
J_{t}^{(i)}\left(x_{t}\right)=\left(y_{t}-x_{t}\right)^{2}+\min _{x_{t-1}}\left[u\left(x_{t-1}-x_{t}\right)^{2}+J_{t-1}^{(i)}\left(x_{t-1}\right)\right], \\
J_{t}^{(k)}\left(x_{t}\right)=\left(y_{t}-x_{t}\right)^{2}+\min _{x_{t-1}}\left[u \Delta^{2}+\tilde{J}_{t-1}\left(x_{t-1}\right)\right] .
\end{gathered}
$$

This implies that each Bellman function has finite-parametric representation. Note also that at each step the number of quadratic functions, needed for the representation of the Bellman function, generally speaking, increases by one. This leads to the fact that the procedure has complexity $O\left(N^{2}\right)$. At the same time, numerous experiments on simulated data showed, that two or three quadratic functions is usually enough to completely represent each Bellman function.

Modification of the above described algorithm is based on the following idea.

It is easy to see that if $\tilde{x}_{1}, \tilde{x}_{2}, \ldots, \tilde{x}_{N}$ is minimum point of the criterion (6), then $\tilde{x}_{t} \in[m, M]$ for each $t=1,2, \ldots, N$, where $m=\min (Y)$ and $M=\max (Y)$. So we can omit all $J_{t}^{(i)}\left(x_{t}\right) \geq \min _{i \neq j}\left[J_{t}^{(j)}\left(x_{t}\right)\right]$ for $x_{t} \in[m, M]$.

Let us denote the number of functions, needed for the representation of the Bellman function at each step, by $m_{1}, m_{2}, \ldots, m_{N}$. It can be shown that if there exists $\quad M=$ const $\quad$ such that $m_{1}^{2} \ln m_{1}^{2}+m_{2}^{2} \ln m_{2}^{2}+\ldots \quad+m_{N}^{2} \ln m_{N}^{2} \leq M \cdot N$, the procedure has the computational complexity $O(N)$.

This procedure can be extended for the case of image analysis by means, for example, of approximation of the initial lattice-like neighborhood graph of variables by the succession of trees or other techniques, described in [1].

\section{Experiments}

For an experimental study of complexity of the algorithm, described in section 3 , the following data model has been used

Observable signal: $Y=\left(y_{t}=x_{t}+\xi_{t}, t=1, \ldots, N\right), y \in R$ where $\xi$ - additive white Gaussian noise, with zero mean and variance $\sigma^{2}$.

Hidden signal: $X=\left(x_{t}, t=1, \ldots, N\right)$, where $x_{1}=z_{1}, x_{t}=x_{t-1}$ with probability $p$ and $x_{t}=z_{t}$ with probability $1-p$ for $t=2, \ldots, N . z_{t}$ are independent uniformly distributed random variables on interval $[0,50]$.

A random distribution of the mean number of quadratic function in the composition of Bellman functions $\left(m_{1}+m_{2}+\ldots+m_{N}\right) / N$ was constructed by MonteCarlo method for $N=100,200,500,1000$. (Fig. 2).

Results of experiments have shown that the procedure has the average complexity $O(N)$. 


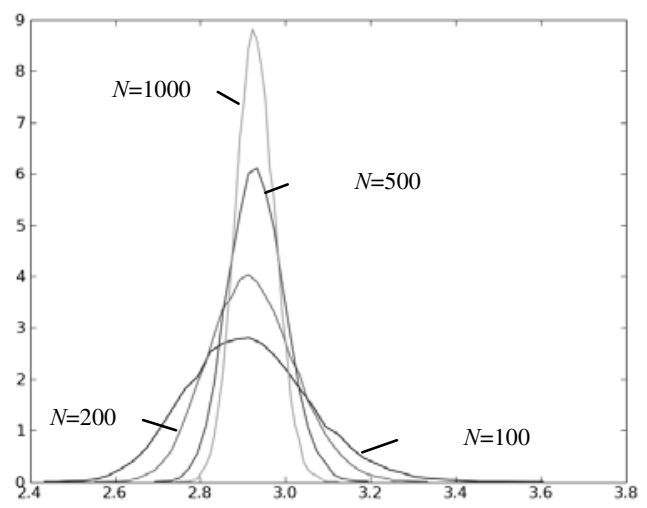

Fig. 2. A random distribution of the mean number of quadratic function in the composition of Bellman functions

\section{Conclusion}

The DP procedure based on parametric family of Bellman functions represented as a minimum of a set of quadratic functions, can reduce the amount of memory, required for its implementation, if the number of parameters of Bellman functions is small enough and less than the amount of memory needed in the case of trivial parameterization by the finite set of values.

This is of particular importance as the number of discrete values of the objective variables makes a massive impact to the requirements of modern $(\min ,+)$ optimization algorithms.

Experimental study let us to guess, that for the vast majority of source data arrays two or three quadratic functions is usually enough to completely represent each Bellman function.

The proposed procedure allows parametric implementation, can take into account a wide range of prior assumptions about the sought-for result, and leads to the effective algorithm of data analysis with linear average computation complexity.

\section{References}

1. Mottl, V., Kopylov, A., Blinov, A., Kostin, A..: Optimization techniques on pixel neighborhood graphs for image processing. In: Graph-Based Representations in Pattern Recognition. Computing, vol. (suppl. 12), pp. 135-145. Springer-Verlag/Wien (1998)

2. Kopylov, A.V.: Parametric dynamic programming procedures for edge preserving in signal and image smoothing. Pattern Recognition and Image Analysis 15(1), 227-230 (2005)

3. Kalman, R.E., Bucy, R.S.: New Results in Linear Filtering and Prediction Theory. Journal of Basic Engineering 83, 95-108 (1961)

4. Szeliski, R., Zabih, R., Scharstein, D., Veskler, O., Kolmogorov, V., Agarwala, A., Tappen, M., Rother, C.: A Comparative Study of Energy Minimization Methods for Markov Random Fields with Smoothness-Based Priors. IEEE Transactions on Pattern Analysis and Machine Intelligence (PAMI) 30(6), 1068-1080 (2008) 